\title{
Mobilités, circulations et frontières. Migrations, mobilités et développement en Afrique
}

\section{Sylvie Ayimpam}

\section{(2) OpenEdition}

\section{Journals}

Édition électronique

URL : https://journals.openedition.org/anthropodev/1068

DOI : $10.4000 /$ anthropodev. 1068

ISSN : 2553-1719

\section{Éditeur}

Presses universitaires de Louvain

Édition imprimée

Date de publication : 1 décembre 2020

Pagination : 193-195

ISBN : 978-2-39061-078-6

ISSN : 2276-2019

\section{Référence électronique}

Sylvie Ayimpam, « Mobilités, circulations et frontières. Migrations, mobilités et développement en Afrique », Anthropologie \& développement [En ligne], 51 | 2020, mis en ligne le 01 décembre 2020, consulté le 31 janvier 2022. URL : http://journals.openedition.org/anthropodev/1068 ; DOI : https:// doi.org/10.4000/anthropodev.1068

La revue Anthropologie \& développement est mise à disposition selon les termes de la Licence Creative Commons Attribution 4.0 International. 


\title{
Mobilités, circulations et frontières. Migrations, mobilités et développement en Afrique
}

\author{
Elieth Eyebiyi et Angèle Mendy (éd.), Niamey/Parakou, Éditions \\ Daraja Press et LASDEL, 2019, 271 p.
}

Sylvie Ayimpam

\begin{abstract}
Valoriser les espaces d'échange entre diverses contrées géographiques ainsi que les multiples opportunités de développement qu'ils présentent dans un contexte de mobilités intra-africaines et extra-africaines, telle est l'ambition de cet ouvrage collectif. Les coordonnateurs de l'ouvrage, Elieth Eyebiyi et Angèle Mendy, ont choisi de questionner la relation entre migrations et développement au regard des défis que posent les nouvelles mobilités en Afrique, en articulant plus spécifiquement ici la question des mobilités à celles des circulations et des frontières. Cet ouvrage est issu d'un cycle de quatre ateliers tenus dans le cadre d'un programme de recherche intitulé Migration, development and regional integration (MIGDEVRI). Ce programme a eu la particularité de réunir des jeunes chercheurs, des intervenants de la société civile et du monde politique, ainsi que des journalistes. L'originalité de cet ouvrage est en premier lieu dans son parti pris d'analyser les différents espaces d'échange et les opportunités de développement qu'ils présentent dans un contexte plus englobant de mobilités intra et extra-africaines, en considérant que la compréhension des migrations africaines induit la compréhension, en miroir, des mobilités dont elles découlent (p. 7). On voit également l'originalité de ce travail dans son choix d'articuler deux thématiques importantes : celle de la relation entre les mobilités, l'éducation et les droits - des questions peu abordées dans les travaux sur les migrations en Afrique -, à celle, plus courante, de la relation entre les frontières et les circulations.
\end{abstract}

En ce qui concerne le domaine de l'éducation, la contribution de Lawrence Okello analyse les mobilités des étudiants africains, en montrant que les études sur ce thème prennent de l'importance au fur et à mesure que croît l'intensité des flux de mobilités d'étudiants entre les pays africains. Auparavant, ces flux partaient le plus souvent des pays africains vers les pays du Nord. L'auteur souligne que l'accroissement de l'intensité de ces migrations étudiantes ne concerne pas que les étudiants, mais également les enseignants, permettant la circulation des savoirs (Okello, p. 115). Zakaria Soré observe pour sa part que ces mobilités des étudiants restent faibles de manière générale, en raison notamment de la survalorisation des diplômes occidentaux, et de la faible coopération entre universités africaines (Soré, p. 120). La question de la protection des Droits de l'homme 
dans le cadre des mobilités, ou encore la protection sociale des travailleurs migrants, sont abordées à travers des études empiriques intéressantes. Les conditions difficiles de séjour, voire d'expulsion, que peuvent éventuellement vivre les travailleurs migrants en Afrique sont illustrées à travers une étude de la mobilité des travailleurs burkinabés en Afrique centrale, et précisément au Gabon et en Guinée équatoriale (Zidnaba et Barkissa). Ces migrations qui datent des années 1970 ont progressivement été mal perçues dans les pays d'accueil, engendrant des préjugés subjectifs selon lesquels l'installation de ces migrants constituerait une menace d' « invasion démographique », de spoliation économique, de perversion sociale, ou de délinquance étrangère. La Guinée équatoriale et le Gabon ont ainsi mis en place des politiques d'expulsion des migrants irréguliers. Elles concernent pour la plupart des cas d'expulsions forcées faisant suite à des arrestations, des emprisonnements, des maltraitances et des spoliations, qui ont contraint à l'abandon dans le pays d'accueil de l'argent épargné, des biens acquis et, dans certains cas, de la famille. Irissa Zidnaba et Nyalo Barkissa Drabo considèrent cela comme de graves atteintes aux droits des travailleurs burkinabés (p. 73-74). Au-delà du Gabon et de la Guinée équatoriale, le Nigeria est également l'un des points importants de convergence des migrations des travailleurs africains car, à l'instar des deux précédents pays, il est considéré comme ayant un niveau de vie plus élevé que d'autres pays. La situation des travailleurs non qualifiés dans la ville de Lagos est ainsi examinée minutieusement par Sylvester Kahol, tandis que Mutiat Titilope aborde la question de la situation des femmes togolaises qui travaillent dans les entreprises de la ville d'lbadan. La dimension transfrontalière et le passé historique commun sont des éléments facilitant la migration des travailleurs ressortissants des pays voisins vers le Nigeria (Kahol, p. 43-45). Mais les défis de cette migration professionnelle ne sont pas pour autant gommés, notamment celui de disposer des papiers autorisant l'exercice professionnel, comme le montre bien Mutiat Titilope pour les travailleuses togolaises à Ibadan (p. 68-69). Elles sont de ce fait non seulement exposées au harcèlement et au travail au noir, mais elles courent également le risque de rapatriements sans préavis en laissant parfois les biens acquis au Nigeria, comme c'est le cas pour les travailleurs burkinabés.

Avec l'augmentation des mobilités transfrontalières et la promotion par les organisations sous-régionales de la libre circulation des personnes et des marchandises, la question des enjeux des circulations transfrontalières occupe une place importante dans l'ouvrage, et elle est abordée sous différents angles. Astadjam Yacouba examine les trajectoires et l'expérience migratoires des ressortissants d'Afrique de l'Ouest dans la ville de Yaoundé au Cameroun. Elle montre que, si l'intégration est tributaire des éléments individuels structurants, notamment la capacité à constituer des réseaux d'appartenance sur place, l'expérience que procure la migration passant par des trajets indirects devient un atout pour le migrant; ce type de migration multiplie et renforce la capacité du migrant à apprendre les langues étrangères, à rechercher un emploi ou à trouver un logement (p. 177-178). En se basant sur la situation du Maroc, Alimou Diallo aborde la question de la figure de l'étranger dans ce pays, qui est généralement considéré comme un espace de transit des candidats africains à la migration vers l'Europe. En considérant le Maroc comme une destination migratoire à part entière, il examine comment le pays gère 
la mobilité et la circulation des étrangers (p. 237-238). La migration quotidienne entre le Bénin et le Niger, est étudiée par Aziz Mossi à partir d'un poste-frontière. En montrant bien que la migration pendulaire constitue la plus grande partie de ces mouvements, Mossi aborde cependant les conditions et les modalités de ces mouvements quotidiens de manière générale, quels qu'en soient les motifs. II montre que cette circulation quotidienne est assurée par des agents des services frontaliers ayant une "culture professionnelle » où les rackets prennent une place importante ; agents qui ont face à eux des usagers favorables à la pratique de la fraude (p. 233). Le transport des marchandises par les " cascadeurs de motos » en Afrique de l'Ouest est examiné par Lopa Ngassou, qui étudie spécifiquement le trajet Nigeria-Tchad-Centrafrique. II décrypte le système sur lequel repose la mobilité transfrontalière de ce groupe d'acteurs assurant le transport des marchandises par motos. Il analyse la manière dont ces acteurs s'organisent pour surmonter les déterminismes officiels, se conformer aux règles de la circulation des biens et des personnes, et déjouer les lois qu'ils estiment allant à l'encontre de leur épanouissement économique (p. 183-185). La diffusion et l'usage du téléphone mobile dans la ville de Lagos au Nigeria et son rôle dans les mobilités urbaines sont examinés par Naluwembe Binaisa (p. 137-138). II montre que la téléphonie mobile facilite les flux de communication et d'information ainsi que la mobilité des acteurs, en dynamisant davantage le secteur commercial dans cette ville-métropole qui est l'un des principaux pôles d'attraction économique et de migrations continues en Afrique subsaharienne.

On peut regretter que certaines sous-régions d'Afrique ne soient pas représentées dans les études de cas, notamment l'Afrique australe et l'Afrique de l'Est qui sont des régions importantes des mobilités intra-africaines, avec des pôles majeurs de convergences migratoires comme les villes de Johannesburg et de Nairobi. On aurait également aimé que l'ouvrage aborde certaines thématiques qui émergent actuellement avec l'intensification des mobilités en Afrique, comme celles des familles transnationales intraafricaines. On peut en revanche souligner que l'ouvrage a relevé un défi important : celui d'éclairer à la fois les dynamiques de l'expérience migratoire, des trajectoires suivies par les migrants et des espaces migratoires à l'intérieur de l'Afrique. 